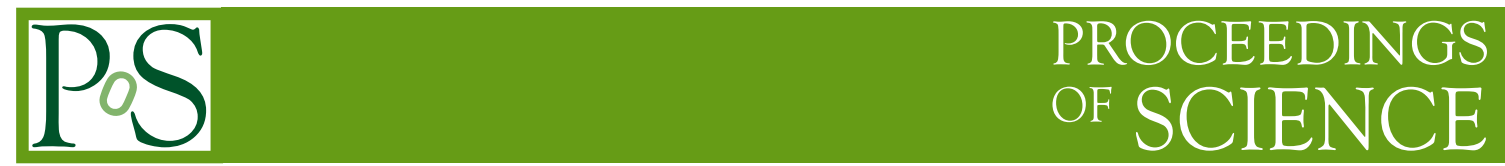

\title{
String Cosmology
}

\section{Marco ZAGERMANN* $*$}

Leibniz University Hannover, Germany

E-mail: Marco.Zagermann@itp.uni-hannover.de

A brief overview of various branches of contemporary string cosmology is given with an emphasis on moduli stabilization, de Sitter vacua, inflation, cosmic strings and primordial gravitational waves.

European Physical Society Europhysics Conference on High Energy Physics July 16-22, 2009

Krakow, Poland

* Speaker.

${ }^{\dagger}$ Work supported by the German Research Foundation (DFG). 


\section{Introduction}

String theory is a promising candidate for a unified and ultraviolet complete description of all particles and interactions, including gravity. The study of its cosmological properties can be motivated in several ways, including

- Completeness: A truly fundamental theory of spacetime, matter and its interactions should be compatible with basic cosmological observations.

- High energy scales: Although one usually associates cosmology with very long wavelength observations, it may directly or indirectly probe energy scales that are out of reach of conventional particle acccelerators. A very good illustration is the UV-sensitivity of slow-roll inflation models, which has several roots:

- The energy scale of inflation may be as high as the GUT scale: $V_{\text {inf }}^{1 / 4} \sim M_{\mathrm{GUT}} \varepsilon^{1 / 4}$, where $\varepsilon \equiv 1 / 2\left(M_{P} V^{\prime} / V\right)^{2} \ll 1$ is the first slow-roll parameter.

- The second slow-roll parameter, $\eta \equiv M_{P}^{2} V^{\prime \prime} / V$, is in general sensitive to even Planck suppressed operators, which therefore have to be known in a given model.

- Detectable primordial tensor modes from inflation require (trans-)Planckian field excursions for the inflaton during inflation, $\Delta \varphi \sim M_{P}$ (see Section 6).

- Certain general features of string theory may have direct implications for cosmology. Examples are the presence of extra dimensions and the moduli fields resulting from their compactification, as well as the ubiquity of various types of extended objects such as D-branes, which could play the rôle of cosmological defects, e.g., in the form of cosmic strings.

As is quite obvious from the above, cosmology probes string theory in a rather complementary way as compared to conventional particle physics, with a corresponding different emphasis of the technical issues that have to be understood. One particularly important technical aspect is the stabilization of moduli fields, without which a quantitative discussion of string cosmology would hardly be possible.

\section{Moduli stabilization}

The simplest string compactifications on spaces such as tori or Calabi-Yau manifolds yield four-dimensional (4D) effective actions that generically comprise a large number of very light or massless scalar fields. These "moduli fields" describe deformations of the background compactification data and can be divided into closed and open string moduli. The former essentially descend from the 6D components of the 10D supergravity fields (which correspond to vibration modes of closed strings), whereas the open string moduli parameterize background data of the D-brane setup in the compact space such as their positions, orientations or gauge field configurations. Although they generally only interact very weakly with Standard Model-like matter fields, the moduli can cause various phenomenological problems, e.g., regarding fifth force experiments, Big Bang Nucleosynthesis or overclosure constraints, depending on their precise mass range. A simple way to avoid all these constraints would be to make the moduli sufficiently heavy (usually well above the $\mathrm{TeV}$ scale), and in order to achive this goal, two principal mechanisms are often discussed: 
- p-form fluxes: Background fluxes of the antisymmetric tensor field strengths (p-forms) of 10D supergravity through p-dimensional cycles of the internal manifold lead to a classical contribution to the effective 4D scalar potential for the moduli that parameterize the deformations of these cycles.

- Quantum corrections: Subleading corrections in the string coupling $g_{s}$ or the inverse string tension $\alpha^{\prime}$ give additional contributions to the scalar potential that are most relevant for those moduli that do not receive a tree-level potential. These moduli are often also used as inflaton candidates, as the subleading nature of their potential may help to keep the potential flat enough for slow-roll inflation.

The interplay of these two mechanisms is presently best understood in type IIB string theory, where so far most work on string cosmology has been done. Much of this work focused on the construction of de Sitter vacua and inflationary model building.

\section{3. de Sitter vacua}

Stabilizing all scalar fields in a positive local minimum of the effective potential can model a Universe with an effective positive cosmological constant. A popular scenario is due to [1], where the moduli are stabilized in a Calabi-Yau orientifold compactification with three-form fluxes and non-perturbative quantum corrections. In this setup, the three-form fluxes stabilize the complex structure (or "shape") moduli as well as the dilaton, whose vacuum expectation value determines the string coupling. The remaining moduli, which receive a potential only at subleading order in $g_{s}$ or $\alpha^{\prime}$, are the Kähler (or "size") moduli, as well as the D3-brane positions in the CalabiYau space. More precisely, in [1] the Kähler moduli, which, in particular, include the overall volume modulus, $\rho$, are stabilized by a non-perturbative contribution to the superpotential induced by gaugino condensation on D7-branes or D3-brane instantons. The resulting superpotential for the volume modulus is of the form $W=W_{0}+A e^{-a \rho}$, where $W_{0}$ is a $\rho$-independent remnant from the flux superpotential, and $A$ and $a$ are constants ${ }^{1}$. Together with the Kähler potential $K=-3 \ln (\rho+$ $\bar{\rho})$, the F-term supergravity potential then leads to a supersymmetric Anti-de Sitter minimum. A potential contribution from a supersymmetry-breaking anti-D3-brane is then suggested in [1] as a means to "uplift" the AdS minimum to a metastable de Sitter vacuum.

A related setup, the "LARGE volume" scenario of [2], also uses the admixture of $\alpha^{\prime}$ corrections.

\section{Inflation}

As mentioned earlier, most inflation scenarios in string theory identify the inflaton with a modulus that receives a potential only at subleading order. A generic problem in these models is that certain other moduli tend to interfere quite severely with slow-roll inflation, because they often form very steep runaway directions of the simplest scalar potentials. This is in particular true

\footnotetext{
${ }^{1}$ More precisely, $A$ generically depends on other moduli (see also Section 4). If these are much heavier than $\rho$, they are usually integrated out, and $A$ is treated as an effectively constant parameter.
} 
for the volume modulus and the dilaton and implies that moduli stabilization and inflation can in general not be discussed separately. Because of this, the most popular arena for building string inflation models has so far been type IIB string theory on Calabi-Yau orientifolds with fluxes. Here the classically light moduli are the D3-brane moduli and the Kähler moduli, whose subleading potentials make these fields popular inflaton candidates. Two classes of inflation models can then be distinguished:

(i) Open string or "brane" inflation models, where the inflaton is a D3-brane position modulus on the Calabi-Yau space

(ii) "Kähler modulus inflation models", where the inflaton is identified with a suitable Kähler (i.e., closed string) modulus or an axionic partner thereof.

The most popular D-brane inflation models include warped D3/D3-brane inflation [3], D3/D7brane inflation [4] and DBI-inflation [5]. Examples for Kähler modulus inflation models include racetrack inflation [6], blow-up modulus inflation [7] or fiber inflation [8].

As an illustration of some of the technical difficulties one may encounter in such models, I describe a few details of the popular warped D3/ $\overline{\mathrm{D} 3}$-brane model. The original idea of this scenario goes back to [9], where the inflaton is identified with the distance between a D3 and a $\overline{\mathrm{D} 3}$-brane in the $6 \mathrm{D}$ compact space with their Coulombic attraction giving rise to the inflaton potential. Unfortunately, this potential generically becomes sufficiently flat only for interbrane separations larger than the actual diameter of the compact space. [3] therefore suggests to put this brane configuration into a warped throat geometry with $6 \mathrm{D}$ metric of the form

$$
d s_{(6)}^{2}=h^{1 / 2}(\varphi)\left[d \varphi^{2}+\varphi^{2} d s_{(5)}^{2}\right] .
$$

Here, $\varphi$ is the distance from the tip of the throat, at which the $\overline{\mathrm{D} 3}$-brane is dynamically fixed by the fluxes, to the D3-brane (whose position is not fixed by the fluxes). $d s_{(5)}^{2}$ denotes the remaining 5D part of the metric, and $h(\varphi)$ is a warp factor that depends on the radial distance and that also appears in front of the 4D spacetime part of the metric. This warp factor redshifts the Coulombic interbrane potential and leads to a suppression of the eta parameter by a factor $h_{0}^{-1}$, where $h_{0}$ is the value of the warp factor at the position of the $\overline{\mathrm{D} 3}$-brane at the tip of the throat. A strongly warped throat with $h_{0} \gg 1$ thus allows for a Coulomb potential compatible with the requirements of slowroll inflation. Moreover, it also has the additional benefit of redshifting the effective tension of the cosmic strings produced after brane annihilation [3] (see also section 5).

Unfortunately, the model just described does not work, because even though the Coulomb potential is flattened by the warping along the brane separation modulus $\varphi$, it has a rapidly decreasing runaway direction along the overall volume modulus $\rho$, so that rapid descent along $\rho$ (i.e. rapid decompactification of the compact manifold!) will prevent any slow-roll along $\varphi$. One thus has to take volume stabilization into account also for the inflationary dynamics of this model. Doing this along the lines of [1] via a non-perturbative F-term potential, however, gives rise to another problem: The volume-stabilizing F-term potential in the presence of the mobile D3-brane also introduces a new $\varphi$-dependence into the potential. The reason is that the non-perturbative superpotential described in the previous section exhibits a dependence on $\varphi$ as $W=W_{0}+A(\varphi) e^{-a \rho}$, whereas the Kähler potential becomes ${ }^{2} K=-3 \ln \left[\rho+\bar{\rho}+|\varphi|^{2}\right]$. The $\varphi$-dependence of the func-

\footnotetext{
${ }^{2}$ A related $\varphi$-dependence also enters the uplift potential that generates a de Sitter vacuum after inflation (see section
} 
tion $A$ has been calculated in [10] from the gravitational backreaction of the D3-brane on the warp factor, and it was found that the scalar potential in general may receive $\varphi^{3 / 2}$-terms from the above mentioned effects, leading to inflection point inflation models. The lesson from this example is that very detailed computational controll may be required in order to assess the cosmological properties of a given inflation model in a UV-complete theory such as string theory.

\section{Cosmic (super)strings}

Brane inflation models such as the warped D3/ $\overline{\mathrm{D} 3}$-brane inflation model of the previous section typically end by a formation of cosmic strings in the form of D1-branes, fundamental strings, $(p, q)$-strings or wrapped Dp-branes with $p>1[11,12]$. A priori these cosmic strings are phenomenologically extremely problematic, but in situations with sufficiently strong warping, the effective cosmic string tension may be lowered to cosmologically acceptable values, and such cosmic strings could then even provide exciting opportunities for future observations. An interesting property of these "cosmic superstrings" is that they may deviate in certain ways from ordinary gauge theory solitons, e.g., regarding their reconnection probability (see e.g. [12]).

\section{Observable tensor modes?}

The Lyth bound [13]

$$
\left(\frac{\Delta \varphi}{M_{P}}\right) \sim\left(\frac{r}{0.01}\right)^{\frac{1}{2}}
$$

relates an observable tensor to scalar ratio, $r$, of the $\mathrm{CMB}$ to a large excursion, $\Delta \varphi$, of the inflaton field during inflation. Planckian field ranges, however, are not so easy to obtain for many string inflation models. In particular, for a D3-brane on a symmetric torus or in a warped throat, the maximal distance it can travel is geometrically limited and translates into strong upper bounds for the possible tensor to scalar ratio, making their observation in these models with near future experiments very unrealistic [14].

Various ways to evade these upper limits for brane inflation have recently been suggested, ranging from wrapped Dp-branes [15], large complex structure limits [16], monodromies [17] or multibrane models [18] to models such as fiber inflation [8] or multi axion field models [19], where the inflaton is no longer a brane modulus. These and similar proposals certainly deserve a closer investigation, especially if tensor modes indeed happen to be observed in the future.

\section{String cosmology beyond type IIB?}

Surprisingly little is known about de Sitter vacua or inflation in string theories other than the type IIB theory. This is a direct consequence of the fact that moduli stabilization has been much more explored for the IIB string. In a sense, though, moduli stabilization is even simpler in type IIA string theory, as the more diverse types of p-form fluxes one may switch on there allow a stabilization of all geometric moduli at tree-level [20], i.e., without the help of quantum

3) [3]. 
corrections as in [1]. In certain cases, one may even stabilize all closed string moduli by fluxes in a parameterically controlled regime where all quantum corrections are negligible [21].

Direct use of these models for cosmology, however, is hampered by quite strong no-go theorems against de Sitter vacua and slow-roll inflation in these purely classical flux compactifications [22]. More precisely, the no-go theorems of [22] state that in a classical type IIA compactification on a Ricci-flat manifold such as a Calabi-Yau space with O6-planes and D6-branes as well as the most general p-form fluxes, the epsilon parameter is bounded from below by $27 / 13$. This theorem follows from simple scaling considerations of the classical potentials that can appear in this setup and which show that the potential is always too steep in the direction of a certain combination of the volume modulus and the dilaton whenever it is positive.

In order to circumvent this no-go theorem, one would either have to go to a regime in which subleading corrections in $g_{s}$ or $\alpha^{\prime}$ are relevant and may change the scaling in the volume-dilaton plane, or/and introduce additional classical features in the compactification setup that have a similar effect. The simplest such classical feature would arguably be a departure from the Ricci-flatnes (i.e. the Calabi-Yau geometry) of the internal space. In fact, this departure would be very natural, as the backreaction of the p-form fluxes on the geometry usually lead to non-Calabi-Yau spaces in the type IIA theory.

In [23], and later in [24], various attempts were made to find de Sitter vacua or inflationary trajectories for well-understood geometries with nontrivial Ricci-curvature, but so far only new no-go theorems or de Sitter extrema with too tachyonic directions could be identified.

The general construction of controlled de Sitter vacua or slow-roll inflation in IIA string theory is still an open problem, and promising directions for improvement might involve either more general non-Calabi-Yau spaces, the use of quantum corrections as, e.g., in [25], or the combination of more general classical ingredients as in [26].

\section{References}

[1] S. Kachru, R. Kallosh, A. D. Linde and S. P. Trivedi, Phys. Rev. D 68 (2003) 046005 [arXiv: hep-th/0301240].

[2] V. Balasubramanian, P. Berglund, J. P. Conlon and F. Quevedo, JHEP 0503 (2005) 007 [arXiv:hep-th/0502058].

[3] S. Kachru, R. Kallosh, A. D. Linde, J. M. Maldacena, L. P. McAllister and S. P. Trivedi, JCAP 0310, 013 (2003) [arXiv: hep-th/0308055].

[4] K. Dasgupta, C. Herdeiro, S. Hirano and R. Kallosh, Phys. Rev. D 65 (2002) 126002 [arXiv:hep-th/0203019].

[5] M. Alishahiha, E. Silverstein and D. Tong, Phys. Rev. D 70 (2004) 123505 [arXiv: hep-th/0404084].

[6] J. J. Blanco-Pillado et al., JHEP 0411 (2004) 063 [arXiv : hep-th / 0406230 ].

[7] S. Krippendorf and F. Quevedo, [arXiv:0901.0683 [hep-th]].

[8] M. Cicoli, C. P. Burgess and F. Quevedo, JCAP 0903 (2009) 013 [arXiv: 0808 . 0691 [hep-th]].

[9] G. R. Dvali and S. H. H. Tye, Phys. Lett. B 450 (1999) 72 [arXiv : hep-ph/9812483 ]. 
[10] D. Baumann, A. Dymarsky, I. R. Klebanov, L. McAllister and P. J. Steinhardt, Phys. Rev. Lett. 99 (2007) 141601 [arXiv: 0705.3837 [hep-th] ]; A. Krause and E. Pajer, JCAP 0807 (2008) 023 [arXiv:0705.4682 [hep-th] ]. D. Baumann, A. Dymarsky, I. R. Klebanov and L. McAllister, JCAP 0801 (2008) 024 [arXiv: 0706.0360 [hep-th] ].

[11] S. Sarangi and S. H. H. Tye, Phys. Lett. B 536 (2002) 185 [arXiv: hep-th/ 0204074 ].

[12] E. J. Copeland, R. C. Myers and J. Polchinski, JHEP 0406 (2004) 013 [arXiv:hep-th/0312067].

[13] D. H. Lyth, Phys. Rev. Lett. 78 (1997) 1861 [arXiv: hep-ph/9606387].

[14] D. Baumann and L. McAllister, Phys. Rev. D 75 (2007) 123508 [arXiv: hep-th/ 0610285 ].

[15] T. Kobayashi, S. Mukohyama and S. Kinoshita, JCAP 0801 (2008) 028 [arXiv: 0708.4285 [hep-th] ]; M. Becker, L. Leblond and S. E. Shandera, Phys. Rev. D 76, 123516 (2007) [arXiv:0709.1170 [hep-th]].

[16] M. Haack, R. Kallosh, A. Krause, A. D. Linde, D. Lüst and M. Zagermann, Nucl. Phys. B 806 (2009) 103 [arXiv:0804.3961 [hep-th]].

[17] E. Silverstein and A. Westphal, Phys. Rev. D 78 (2008) 106003 [arXiv: 0803.3085 [hep-th] ]; L. McAllister, E. Silverstein and A. Westphal, [arXiv:0808.0706 [hep-th]].

[18] K. Becker, M. Becker and A. Krause, Nucl. Phys. B 715 (2005) 349 [arXiv: hep-th/0501130].

[19] S. Dimopoulos, S. Kachru, J. McGreevy and J. G. Wacker, JCAP 0808 (2008) 003 [arXiv:hep-th/0507205].

[20] T. W. Grimm and J. Louis, Nucl. Phys. B 718, 153 (2005) [arXiv: hep-th/ 0412277 ]; S. Kachru and A. K. Kashani-Poor, JHEP 0503, 066 (2005) [arXiv : hep-th/ 0411279 ].

[21] O. DeWolfe, A. Giryavets, S. Kachru and W. Taylor, JHEP 0507, 066 (2005) [arXiv: hep-th/0505160].

[22] M. P. Hertzberg, S. Kachru, W. Taylor and M. Tegmark, JHEP 0712, 095 (2007) [arXiv:0711.2512 [hep-th]].

[23] S. S. Haque, G. Shiu, B. Underwood and T. Van Riet, Phys. Rev. D 79, 086005 (2009) [arXiv:0810.5328 [hep-th] ]; C. Caviezel, P. Koerber, S. Körs, D. Lüst, T. Wrase and M. Zagermann, JHEP 0904, 010 (2009) [arXiv: 0812 . 3551 [ hep-th] ]; R. Flauger, S. Paban, D. Robbins and T. Wrase, Phys. Rev. D 79, 086011 (2009) [arXiv:0812.3886 [hep-th]].

[24] D. Roest, Class. Quant. Grav. 26 (2009) 135009 [arXiv:0902.0479 [hep-th]]; G. Dall'Agata, G. Villadoro and F. Zwirner, [arXiv:0906.0370 [hep-th] ]; U. H. Danielsson, S. S. Haque, G. Shiu and T. Van Riet, JHEP 0909, 114 (2009) [arXiv: 0907.2041 [hep-th] ]; B. de Carlos, A. Guarino and J. M. Moreno, [arXiv:0907.5580 [hep-th] ].

[25] E. Palti, G. Tasinato and J. Ward, JHEP 0806, 084 (2008) [arXiv: 0804.1248 [hep-th] ].

[26] E. Silverstein, Phys. Rev. D 77, 106006 (2008) [arXiv:0712.1196 [hep-th] ]. 